\title{
Various Empirical Equations to Unify between the Gravitational Force and the Electromagnetic Force
}

\author{
Tomofumi Miyashita \\ Miyashita Clinic, Osaka, Japan \\ Email: tom_miya@plala.or.jp
}

How to cite this paper: Miyashita, T. (2021) Various Empirical Equations to Unify between the Gravitational Force and the Electromagnetic Force. Journal of Modern Physics, $12,859-869$.

https://doi.org/10.4236/jmp.2021.127054

Received: April 3, 2021

Accepted: May 18, 2021

Published: May 21, 2021

Copyright $\odot 2021$ by author(s) and Scientific Research Publishing Inc. This work is licensed under the Creative Commons Attribution International License (CC BY 4.0).

http://creativecommons.org/licenses/by/4.0/

\section{(c) (i) Open Access}

\begin{abstract}
Previously, we proposed several empirical equations to describe the relationship between an electromagnetic force and the temperature of the cosmic microwave background (CMB).We attempted to justify why our empirical equations cannot be coincidental from the mathematical connections between our three equations. However, there are small errors in our empirical equations, which may lead to "indeed or not" arguments. After evaluating our equations, we discovered a method to improve the accuracy of the numerical calculations. For the value of the CMB, we used $2.72642 \mathrm{~K}$ instead of $2.72548 \mathrm{~K}$. Regarding the factor of $9 / 2$, we used 4.48870 instead of 4.5 . Regarding the factor of $\pi$, we used 3.13189 instead of 3.14159. Then, the error becomes less than $10^{-5}$. This means that our equations cannot be coincidental. Furthermore, we attempt to provide hints on how to construct the background theory.
\end{abstract}

\section{Keywords}

Temperature of the Cosmic Microwave Background, Wagner's Equation, Jarzynski's Equality

\section{Introduction}

Our main research explains the current independent constant voltage loss $(0.35$ V) in Sm doped Ceria (SDC) electrolytes in solid oxide fuel cells (SOFC). This voltage loss cannot be explained by Wagner's equation. During the oxygen ion hopping conduction with enough electron's atmosphere, this voltage loss may be explained by Jarzynski's equality. The discovered equivalent circuit is different from the usual RC circuit, and seems to be useful to explain the gravitational force. Then, we discovered Equations (1)-(3) [1] [2] and [3]. 
Previously, we reported the empirical equation for a gravitational force in terms of the temperature of the cosmic microwave background (CMB) [1] [2]:

$$
\frac{G m_{p}}{\frac{\lambda_{p}}{2}} \times 1 \mathrm{~kg}=\frac{9}{2} k T_{c}
$$

where $G, m_{p}, \lambda_{p}, k$, and $T_{c}$ are the gravitational constant, the rest mass of a proton, the Compton wavelength, the Boltzmann constant and the temperature of the $\mathrm{CMB}$, respectively. One kilogram is the standard unit of mass, as previously explained [1]. The error was $0.217 \%$.

Below, we present the empirical equation for the ratio between a gravitational force and an electric force [3]:

$$
\frac{G m_{p}^{2}}{\frac{e^{2}}{4 \pi \varepsilon_{0}}}=4.5 \times \frac{m_{e}}{e} \times \hbar c \times\left(1 \frac{\mathrm{C}}{\mathrm{J} \cdot \mathrm{m}} \times \frac{1}{1 \mathrm{~kg}}\right)
$$

where $e, \varepsilon_{0}, m_{e}, \hbar$ and $c$ are the electric charge of one electron, the electric constant, the rest mass of an electron, the reduced Planck constant and the speed of light, respectively. As previously noted, $1 \mathrm{C} / \mathrm{J} / \mathrm{m}$ is the standard electrostatic quantity [3]. The error was only $0.0578 \%$.

Below, we present that Coulomb's law with distance can be expressed in terms of the CMB [3].

$$
\frac{e^{2}}{4 \pi \varepsilon_{0} r^{2}}=\frac{1}{r^{2}} \frac{e \pi}{m_{e} c^{2}} k T_{c} \times\left(1 \frac{\mathrm{J} \cdot \mathrm{m}}{\mathrm{C}}\right)
$$

where $r$ is the distance between two electrons. The error was $0.274 \%$.

We tried to explain Equation (1), theoretically [1] [2]. However, after the discoveries of empirical Equations (2) and (3), we noticed that there were confusions in our definitions between the Gibbs volume entropy and PV ( $\mathrm{P}$ is pressure and $\mathrm{V}$ is volume). To elucidate the equivalent circuit, there need the more difficult thermodynamic concept. So, we abandoned the theoretical explanation for Equations (2) and (3) [3].

Our conclusion in the previous report was that the mathematical connections among Equations (1)-(3) provide evidence that they are not coincidental [3]. There remain following two main problems in our previous works.

Problem1: We could not explain three equations theoretically.

It means that we cannot provide the suitable equivalent circuit in our equations. The equivalent circuit may be different from the usual LRC circuits. There may be the strong combinations with the difficult advanced thermodynamics, which include the inevitable dissipation.

Problem 2: There are small errors in our empirical equations

Equations (1)-(3) are not complex and connected each other. But there remains the possibility that the small errors may lead to "indeed or not" arguments.

In this report, we searched for a compensation method to solve the above Problem 2 and to provide hints to solve Problem 1. The rest of the paper is orga- 
nized as follows. In Section 2, we present the symbol list, the calculation results for frequently used values and important equations. In Section 3, we present new expressions for Equations (1)-(3). In Section 4, we present the newly discovered empirical equations. In Section 5, we present our compensation methods and the verification of newly discovered empirical equations. In Section 6, we attempt to provide hints on how to construct a background theory.

\section{Symbol List and Frequently Used Values}

\subsection{Symbol List}

These values were obtained from Wikipedia.

$G$ : gravitational constant: $6.6743 \times 10^{-11}\left(\mathrm{~m}^{3} \cdot \mathrm{kg}^{-1} \cdot \mathrm{s}^{-2}\right)$

$T_{c}$ : temperature of the cosmic microwave background: $2.72548(\mathrm{~K})$

$k$. Boltzmann constant: $1.380649 \times 10^{-23}\left(\mathrm{~J} \cdot \mathrm{K}^{-1}\right)$

c. speed of light: $299,792,458(\mathrm{~m} / \mathrm{s})$

h: Planck constant: $6.62607015 \times 10^{-34}(\mathrm{Js})$

$\hbar$ : Dirac constant (reduced Planck constant): $1.054571817 \times 10^{-34}(\mathrm{Js})$

$\mathcal{E}_{0}$ : electric constant: $8.8541878128 \times 10^{-12}\left(\mathrm{~N} \cdot \mathrm{m}^{2} \cdot \mathrm{C}^{-2}\right)$

$\mu_{0}$ : magnetic constant: $1.25663706212 \times 10^{-6}\left(\mathrm{~N} \cdot \mathrm{A}^{-2}\right)$

$e$ electric charge of one electron: $-1.602176634 \times 10^{-19}(\mathrm{C})$

$q_{m}$ : magnetic charge of one magnetic monopole: $4.13566770 \times 10^{-15}(\mathrm{~Wb})$

(this value is only a theoretical value, $q_{m}=h / e$ )

$m_{p}$ : rest mass of a proton: $1.672621923 \times 10^{-27}(\mathrm{~kg})$

$m_{e}:$ rest mass of an electron: $9.1093837 \times 10^{-31}(\mathrm{~kg})$

$\lambda_{p}$ : Compton wavelength for a proton: $1.32141 \times 10^{-15}(\mathrm{~m})$

$\lambda_{e}$ : Compton wavelength for an electron: $2.4263102367 \times 10^{-12}(\mathrm{~m})$

$r_{e}$ : classic electron radius: $2.8179403227 \times 10^{-15}(\mathrm{~m})$

$a_{0}$ : Bohr radius: $0.529177210 \times 10^{-10}(\mathrm{~m})$

$R_{\infty}$ : Rydberg constant: 10,973,731.568 $\left(\mathrm{m}^{-1}\right)$

$\alpha$ : fine-structure constant: $1 / 137.0359991$

$R_{k}$ : von Klitzing constant: 25,812.80745 $(\Omega)$

$Z_{0}$ : wave impedance in free space: $376.730313668(\Omega)$

\subsection{Calculation Results for the Frequently Used Values}

The calculation results for several frequently used values are presented in this section. The number of significant figures used is 5 .

$$
\begin{gathered}
\frac{e^{2}}{4 \pi \varepsilon_{0}}=\frac{\left(1.6022 \times 10^{-19}\right)^{2}}{4 \pi \times 8.8542 \times 10^{-12}}=2.3071 \times 10^{-28}(\mathrm{~J} \cdot \mathrm{m}) \\
\frac{e}{4 \pi \varepsilon_{0}}=\frac{1.6022 \times 10^{-19}}{4 \pi \times 8.8542 \times 10^{-12}}=1.4400 \times 10^{-9}(\mathrm{~J} \cdot \mathrm{m} / \mathrm{C}) \\
\frac{e^{2}}{4 \pi \varepsilon_{0}} \frac{e}{4 \pi \varepsilon_{0}}=2.3071 \times 10^{-28} \times 1.4400 \times 10^{-9}=3.3221 \times 10^{-37}\left(\mathrm{~J}^{2} \cdot \mathrm{m}^{2} \cdot \mathrm{C}^{-1}\right)
\end{gathered}
$$




$$
\begin{aligned}
& G m_{p}^{2}=6.6743 \times 10^{-11} \times\left(1.6726 \times 10^{-27}\right)^{2}=1.8672 \times 10^{-64}(\mathrm{~J} \cdot \mathrm{m}) \\
& G m_{e}^{2}=6.6743 \times 10^{-11} \times\left(9.1093 \times 10^{-31}\right)^{2}=5.5384 \times 10^{-71}(\mathrm{~J} \cdot \mathrm{m})
\end{aligned}
$$

When $T_{c}$ is $2.7264 \mathrm{~K}$,

$$
k T_{c}=1.3807 \times 10^{-23} \times 2.7264=3.7642 \times 10^{-23}(\mathrm{~J})
$$

\subsection{Important Equations}

The important equations are presented in this section.

$$
\begin{aligned}
& \frac{e^{2}}{4 \pi \varepsilon_{0} \hbar c}=\alpha=\frac{1}{137.036} \\
& \frac{q_{m}^{2}}{\mu_{0} \pi \hbar c}=\frac{1}{\alpha}=137.036 \\
& \hbar c=\left(\frac{e^{2}}{4 \pi \varepsilon_{0}}\right)^{\frac{1}{2}}\left(\frac{q_{m}^{2}}{\mu_{0} \pi}\right)^{\frac{1}{2}}
\end{aligned}
$$

\section{New Expressions for Equations (1)-(3)}

The mathematical connections among Equations (1)-(3) have already been proven [3]. The purpose of this section is to explain newly discovered equations in Section 4.

\subsection{New Expression for Equation (1)}

For convenience, Equation (1) is rewritten as Equation (13).

$$
\frac{G m_{p}}{\frac{\lambda_{p}}{2}}=\frac{9}{2} k T_{c}
$$

Here,

$$
\lambda_{p}=\frac{h}{m_{p} c}
$$

From Equations (13) and (14),

$$
\frac{G m_{p} m_{p} c^{2}}{h c}=\frac{9}{4} k T_{c}
$$

From Equations (12) and (15),

$$
G m_{p}^{2}=\frac{4.5 \pi}{c^{2}}\left(\frac{e^{2}}{4 \pi \varepsilon_{0}}\right)^{\frac{1}{2}}\left(\frac{q_{m}^{2}}{\mu_{0} \pi}\right)^{\frac{1}{2}} k T_{c}
$$

\subsection{New Expression for Equation (2)}

For convenience, Equation (2) is rewritten as Equation (17). 


$$
\frac{G m_{p}^{2}}{\frac{e^{2}}{4 \pi \varepsilon_{0}}}=4.5 \times \frac{m_{e}}{e} \times \hbar c
$$

From Equations (12) and (17),

$$
G m_{p}^{2}=4.5 \times \frac{m_{e}}{e} \times\left(\frac{e^{2}}{4 \pi \varepsilon_{0}}\right)^{\frac{3}{2}}\left(\frac{q_{m}^{2}}{\mu_{0} \pi}\right)^{\frac{1}{2}}
$$

\subsection{New Expression for Equation (3)}

From Equation (3),

$$
\frac{m_{e} c^{2}}{e \pi} \times \frac{e^{2}}{4 \pi \varepsilon_{0}}=k T_{c}
$$

\section{Our Newly Discovered Empirical Equations}

\subsection{Empirical Equation between the Rest Mass Values of an Electron and a Proton}

The empirical equation between the rest mass values of a proton and an electron is presented in Equation (20). Using Equation (20), we discovered three empirical equations with the rest mass of a proton. And we discovered three empirical equations with the rest mass of an electron.

$$
\begin{gathered}
\frac{m_{p}}{m_{e}}=\frac{1}{4.5 \times \pi} \frac{q_{m}}{e} \\
\frac{m_{p}}{m_{e}}=\frac{1.6726 \times 10^{-27}}{9.1094 \times 10^{-31}}=1836.2 \\
\frac{1}{4.5 \times \pi} \frac{q_{m}}{e}=\frac{1}{4.5 \times 3.1416} \times \frac{4.1357 \times 10^{-15}}{1.6022 \times 10^{-19}}=1825.9 \\
\text { Error }=\frac{1836.2}{1825.9}-1=0.00563
\end{gathered}
$$

\subsection{Three Empirical Equations with the Rest Mass of a Proton}

From Equation (16),

$$
G m_{p}^{2}=\frac{4.5}{2} \frac{e q_{m}}{c} k T_{c}
$$

From Equations (18) and (20),

$$
G m_{p}^{2}=4.5 \times 4.5 \times \pi \frac{m_{p}}{q_{m}} \times\left(\frac{e^{2}}{4 \pi \varepsilon_{0}}\right)^{\frac{3}{2}}\left(\frac{q_{m}^{2}}{\mu_{0} \pi}\right)^{\frac{1}{2}}
$$

Therefore,

$$
G m_{p}=4.5 \times 4.5 \times \frac{e c}{2} \times\left(\frac{e^{2}}{4 \pi \varepsilon_{0}}\right)
$$

From Equations (19) and (20), 


$$
4.5 \times \frac{m_{p} c^{2}}{q_{m}} \times\left(\frac{e^{2}}{4 \pi \varepsilon_{0}}\right)=k T_{c}
$$

\subsection{Three Empirical Equations with the Rest Mass of an Electron}

From Equations (20) and (24),

$$
G\left(\frac{q_{m}}{e} \times \frac{m_{e}}{4.5 \pi}\right)^{2}=\frac{4.5}{2} \frac{e q_{m}}{c} k T_{c}
$$

Therefore,

$$
G m_{e}^{2}=\frac{4.5^{3}}{2} \frac{e^{3}}{q_{m} c} \pi^{2} k T_{c}
$$

From Equations (20) and (26),

$$
G \frac{q_{m}}{e} \times \frac{m_{e}}{4.5 \pi}=4.5 \times 4.5 \times \frac{e c}{2} \times\left(\frac{e^{2}}{4 \pi \varepsilon_{0}}\right)
$$

Hence,

$$
G m_{e}=4.5^{3} \times \pi \times \frac{e^{2} c}{2 q_{m}} \times\left(\frac{e^{2}}{4 \pi \varepsilon_{0}}\right)
$$

For convenience, Equation (19) is rewritten as Equation (32).

$$
\frac{m_{e} c^{2}}{e \pi} \times \frac{e^{2}}{4 \pi \varepsilon_{0}}=k T_{c}
$$

\section{Our Compensation Methods}

In section 3, we propose seven empirical equations. They are Equations ((20), (24), (26), (27), (29), (31) and (32)). Regarding the factor of 9/2, we used 4.48870 instead of 4.5 which was determined from Equation (26). Regarding the factor of $\pi$, we used 3.13189 instead of 3.14159, which was determined from Equations ((20) and (31)). Regarding the value of the CMB, we used $2.72642 \mathrm{~K}$ instead of $2.72548 \mathrm{~K}$, which was determined from Equations ((24), (27), (29) and (32)).

\subsection{Verification of Equation (20)}

From Equation (20) with the compensation method,

$$
\frac{m_{p}}{m_{e}}=\frac{1}{4.4887 \times 3.1319} \times \frac{4.1357 \times 10^{-15}}{1.6022 \times 10^{-19}}=1836.2
$$

Equation (33) is equal to Equation (21). Therefore, the compensation method is perfect.

\subsection{Verification of Equation (24)}

For convenience, Equation (9) is rewritten as Equation (34).

$$
k T_{c}=1.3807 \times 10^{-23} \times 2.7264=3.7642 \times 10^{-23}(\mathrm{~J})
$$

From Equations (24) and (34) with the compensation method, 


$$
\frac{4.4887}{2} \frac{1.6022 \times 10^{-19} \times 4.1357 \times 10^{-15}}{2.9979 \times 10^{8}} 3.7642 \times 10^{-23}=1.8672 \times 10^{-64}
$$

Equation (35) is equal to Equation (7). Therefore, the compensation method is perfect.

\subsection{Verification of Equation (26)}

$$
G m_{p}=6.6743 \times 10^{-11} \times 1.6726 \times 10^{-27}=1.1164 \times 10^{-37}
$$

For convenience, Equation (4) is rewritten as Equation (37).

$$
\frac{e^{2}}{4 \pi \varepsilon_{0}}=\frac{\left(1.6022 \times 10^{-19}\right)^{2}}{4 \pi \times 8.8542 \times 10^{-12}}=2.3071 \times 10^{-28}(\mathrm{~J} \cdot \mathrm{m})
$$

From Equations (26) and (37) with the compensation method,

$$
4.4887^{2} \times \frac{1.6022 \times 10^{-19} \times 2.9979 \times 10^{8}}{2} \times 2.3071 \times 10^{-28}=1.1164 \times 10^{-37}
$$

Equation (36) is equal to Equation (38). Therefore, the compensation method is perfect.

\subsection{Verification of Equation (27)}

From Equation (27) with the compensation method,

$$
4.4887 \times \frac{1.6726 \times 10^{-27} \times\left(2.9979 \times 10^{8}\right)^{2}}{4.1357 \times 10^{-15}} \times 2.3071 \times 10^{-28}=3.7642 \times 10^{-23}
$$

Equation (39) is equal to Equation (9. Therefore, the compensation method is perfect.

\subsection{Verification of Equation (29)}

From Equation (29) with the compensation method,

$$
\begin{aligned}
& \frac{4.4887^{3}}{2} \frac{\left(1.6022 \times 10^{-19}\right)^{3}}{4.1357 \times 10^{-15} \times 2.9979 \times 10^{8}} \times 3.1319^{2} \times 3.7642 \times 10^{-23} \\
& =5.5384 \times 10^{-71}
\end{aligned}
$$

Equation (40) is equal to Equation (8). Therefore, the compensation method is perfect.

\subsection{Verification of Equation (31)}

$$
G m_{e}=6.6743 \times 10^{-11} \times 9.1094 \times 10^{-31}=6.0799 \times 10^{-41}
$$

From Equation (31) with the compensation method,

$$
\begin{aligned}
& 4.4887^{3} \times 3.1319 \times \frac{\left(1.6022 \times 10^{-19}\right)^{2} \times 2.9979 \times 10^{8}}{2 \times 4.1357 \times 10^{-15}} \times 2.3071 \times 10^{-28} \\
& =6.0799 \times 10^{-41}
\end{aligned}
$$

Equation (41) is equal to Equation (42). Therefore, the compensation method 
is perfect.

\subsection{Verification of Equation (32)}

From Equation (32) with the compensation method,

$$
\frac{9.1094 \times 10^{-31} \times\left(2.9979 \times 10^{8}\right)^{2}}{1.6022 \times 10^{-19} \times 3.1319} \times 2.3071 \times 10^{-28}=3.7642 \times 10^{-23}
$$

Equation (43) is equal to Equation (9). Therefore, the compensation method is perfect.

\section{Discussion}

With only three compensation values, we explained 7 equations perfectly. Therefore, we can justify why the discovered compensation values should not coincide. We attempt to provide hints to construct a background theory.

\subsection{Consideration of Our Compensation Method}

Regarding the value of the CMB, we used $2.72642 \mathrm{~K}$ instead of $2.72548 \mathrm{~K}$. This does not indicate experimental measurement error. Our compensation methods use 4.48870 instead of the factor 4.5. Furthermore, regarding the factor of $\pi$, we used 3.13189 instead of 3.14159. This small deviation may be due to unknown theoretical reasons.

For convenience, Equation (20) is rewritten as Equation (44).

$$
\frac{m_{p}}{m_{e}}=\frac{1}{4.5 \times \pi} \frac{q_{m}}{e}
$$

Here,

$$
4.5 \times \pi=14.13716694
$$

The accurate equation should be

$$
\frac{q_{m}}{e} \times \frac{m_{e}}{m_{p}}=14.05809432
$$

Therefore, the two compensation values cannot be independent. Here,

$$
\begin{gathered}
\frac{q_{m}}{e}=R_{k} \\
R_{k} \alpha=\frac{1}{2} \sqrt{\frac{\mu_{0}}{\varepsilon_{0}}}=\frac{Z_{0}}{2}
\end{gathered}
$$

Therefore, we believe that the two compensation values should be related to 137.036. However, we cannot discover an accurate relationship. Perhaps there needs a computer program that can perform vector analysis. With our methods, vector analysis cannot be performed.

\subsection{Consideration of the Relationship with the Jarzynski Equality}

A nonequilibrium work relation is described by the Jarzynski equality [4]. 


$$
\left\langle\mathrm{e}^{-\beta W}\right\rangle=\mathrm{e}^{-\beta \Delta F_{T}}
$$

where $\beta$ is $1 / k T$ and $W$ and $\Delta F_{T}$ are the work and the difference in the Helmholtz free energy, respectively. The angular brackets $\langle-\rangle$ indicate an ensemble of realizations of the process. For adiabatic processes, $\Delta F_{T}$ is zero. The following equation should be added [4]:

$$
\left\langle\mathrm{e}^{-\beta X}\right\rangle=1
$$

where $X$ is the energy in the same adiabatic process and ensemble of realizations. According to Jarzynski, quantum theory can be explained with Equation (50). Hence, $X$ is investigated in detail without background theory. In Equation (50), the temperature ( $T$ ) may be equal to the CMB. Previously, we attempted to explain Equation (1) [1] [2]. However, after the discovery of many empirical equations, we strongly feel that $X$ may be separated into electromagnetic potential energy and kinetic energy, including the rest mass energy.

\subsection{Relationship between $G$ and $k T_{c}$}

In our four empirical equations, we used the gravitational constant $(G)$. We noticed that $G$ is not independent of the CMB. From Equation (26),

$$
m_{p}=4.5 \times 4.5 \times \frac{e c}{2 G} \times\left(\frac{e^{2}}{4 \pi \varepsilon_{0}}\right)
$$

From Equations (24) and (51),

$$
G\left[4.5 \times 4.5 \times \frac{e c}{2 G} \times\left(\frac{e^{2}}{4 \pi \varepsilon_{0}}\right)\right]^{2}=\frac{4.5}{c} \frac{e q_{m}}{2} k T_{c}
$$

Hence,

$$
\frac{4.5^{3}}{2 q_{m}} e c^{3}\left(\frac{e^{2}}{4 \pi \varepsilon_{0}}\right)^{2}=G k T_{c}
$$

From Equation (54) with the compensation method,

$$
\begin{aligned}
& \frac{4.4887^{3}}{2 \times 4.1357 \times 10^{-15}} \times 1.6022 \times 10^{-19} \times\left(2.9979 \times 10^{8}\right)^{3} \times\left(2.3071 \times 10^{-28}\right)^{2} \\
& =2.5124 \times 10^{-33} \\
& \quad G k T_{c}=6.6743 \times 10^{-11} \times 3.7642 \times 10^{-23}=2.5124 \times 10^{-33}
\end{aligned}
$$

Equation (54) is equal to Equation (55). Therefore, the compensation method is perfect. Equation (53) is a little similar with Hawking radiation. Therefore, the relationship between Hawking radiation and Equation (53) should be examined.

\subsection{Consideration for the Four Special Lengths}

About for four special lengths (Classical electron radius, Compton wavelength for an electron, Bohr radius and Rydberg constant), the following Equation (56) is well known. 


$$
r_{e} 2 \pi=\alpha \lambda_{e}=\alpha^{2} a_{0} 2 \pi=\alpha^{3} \frac{1}{2 R_{\infty}}
$$

When $\pi$ is changed from 3.14159 to 3.13189 in Equation (56), the relationship between four lengths seemed to be changed. But there are no problems. We used Equation (4). For convenience, Equation (4) is rewritten below.

$$
\frac{e^{2}}{4 \pi \varepsilon_{0}}=\frac{\left(1.6022 \times 10^{-19}\right)^{2}}{4 \pi \times 8.8542 \times 10^{-12}}=2.3071 \times 10^{-28}(\mathrm{~J} \cdot \mathrm{m})
$$

Then, in Equation (57), we used 3.14159 as $\pi$.

$$
r_{e}=\frac{e^{2}}{4 \pi \varepsilon_{0}} \frac{1}{m_{e} c^{2}}
$$

So, the value of $r_{e}$ cannot be changed. Consequently, Equation (56) can be unchanged.

\subsection{Consideration for the Degree of Freedom outside the Lorenz Invariant Scheme}

The factor of 4.5 and $\pi$ were considered to be related with the degree of freedom inside a proton and an electron [3]. So, our compensations in this report are related with the degree of freedom outside the Lorenz invariant scheme. The degree of freedom inside an electron seems to be $2 \pi$. We thought that $2 \pi$ is related with a spin of electron. Angrick et al. confirmed that the spin of electron cannot be ignored thermodynamically [5]. Furthermore, Aquino et al. discovered the new method for vector analysis [6]. We hope that the degree of freedom outside the Lorenz invariant scheme will be clarified experimentally in detail.

\section{Conclusions}

Previously, we discovered an empirical equation (Equation (1)) relating a gravitational force and the CMB [1]. Next, we discovered the empirical equation (Equation (2)) for the ratio between a gravitational force and an electric force. Coulomb's law with distance can be expressed in terms of the CMB (Equation (3)). Our conclusion was that the mathematical connections among Equations (1)-(3) provide evidence that they are not coincidental [3]. However, there are small errors in our empirical equations, which may lead to "indeed or not" arguments.

Therefore, we attempted to reduce the errors. For this purpose, we searched for an empirical equation between the remaining mass values of an electron and a proton (Equation (20)). Next, we discovered three empirical equations (Equations ((24), (26) and (27)) with the rest mass of a proton. We then discovered three empirical equations (Equations ((29), (31) and (32)) with the rest mass of an electron.

Then, we discovered the compensation methods. For the value of the CMB, we used $2.72642 \mathrm{~K}$ instead of $2.72548 \mathrm{~K}$. However, we felt that this was not the result of experimental measurement errors. This small deviation may be attri- 
buted to unknown theoretical reasons. Regarding the factor of $9 / 2$, we used 4.48870 instead of 4.5. Regarding the factor of $\pi$, we used 3.13189 instead of 3.14159. Then, the errors in the seven equations were less than $10^{-5}$. Therefore, we can justify why the discovered compensation values should not coincide.

Furthermore, we attempted to provide hints on how to construct a background theory. $\mathrm{X}$ in the Jarzynski equality is investigated in quantum physics. $\mathrm{X}$ may be separated into electromagnetic potential energy and kinetic energy, including the rest mass energy. We noted that $\mathrm{G}$ is not independent of the CMB. Equation (53) is similar with Hawking radiation. Therefore, the relationship between Hawking radiation and Equation (54) should be examined. The relationship between four special lengths can be unchanged. The degree of freedom outside the Lorenz invariant scheme is important to explain our compensations.

\section{Conflicts of Interest}

The author declares no conflicts of interest regarding the publication of this paper.

\section{References}

[1] Miyashita, T. (2020) Journal of Modern Physics, 11, 1180-1192. https://doi.org/10.4236/jmp.2020.118074

[2] Miyashita, T. (2020) Journal of Modern Physics, 11, 1159-1160. https://doi.org/10.4236/jmp.2020.1110096

[3] Miyashita, T. (2021) Journal of Modern Physics, 12; Will Be Published.

[4] Jarzynski, C. (2017) Physical Review X, 7, Article ID: 011008. https://doi.org/10.1103/PhysRevX.7.011008

[5] Angrick. C., Braun, J., Ebert, H. and Donath, M. (2021) Journal of Physics. Condensed Matter, 33, Article ID: 115501. https://doi.org/10.1088/1361-648X/abd338

[6] Aquino, F.W. and Wong, B.M. (2018) Journal of Physical Chemistry Letters, 9, 64566462. https://doi.org/10.1021/acs.jpclett.8b02786

\section{Appendix}

The possible problem was discovered in Equation (26) during typesetting for publication. Equation (26) may be the following Equation (59).

$$
G m p=4.5 \times 4.5 \times \frac{e c}{2} \times \frac{\pi}{3.14159} \times\left(\frac{e^{2}}{4 \pi \varepsilon_{0}}\right)
$$

Then, the calculated kTc cannot be determined. 